\title{
Occurrence of occult CSF leaks during standard FESS procedures*
}

\author{
Sarina Bucher ${ }^{1 \#}$, Anton Kugler ${ }^{1,2 \#}$, Elisabeth Probst ${ }^{3}$, Lorenz Epprecht ${ }^{1}$, \\ Rafael R. Stadler', David Holzmann' ${ }^{1}$, Michael B. Soyka' \\ ' Department of Otorhinolaryngology Head and Neck Surgery, University of Zurich and University Hospital Zurich, Zurich, \\ Switzerland \\ ${ }^{2}$ Department of Otorhinolaryngology Head and Neck Surgery, Paracelsus Medical University of Salzburg, Austria \\ ${ }^{3}$ Department of Immunology, University of Zurich and University Hospital Zurich, Zurich, Switzerland
}

Rhinology 56; 2: 166-171, 2018

https://doi.org/10.4193/Rhin17.117

*Received for publication:

June 1, 2017

Accepted: September 9, 2017

"both authors contributed equally

Objective: To determine the incidence of occult cerebrospinal fluid leaks (CSF) after functional endoscopic sinus surgery (FESS) and to evaluate the diagnostic performance of $\beta 2$-transferrin in blood-contaminated conditions.

Study Design: Prospective cohort study.

Methods: An analysis of 57 intraoperative samples using hydrogel $6 \beta 2$-transferrin assay after FESS was undertaken. In case of CSF positive samples and continuing rhinorrhea, reanalysis after $>1$ year was conducted. In-vivo analysis of a primary spontaneous CSF leak sample took place to verify difficulties in detecting $\beta 2$-transferrin in blood-contaminated settings. Own titrations were performed to evaluate detection limits of CSF by $\beta 2$-transferrin and $\beta$-trace protein assays in these settings.

Results: An incidence of $13 \%$ for occult CSF leaks after FESS was found. In blood-contaminated conditions, routine $\beta 2$-transferrin assays showed low sensitivity. In $>1$ year follow-up, all samples were negative for CSF and none of them developed clinical relevant CSF leaks or meningitis.

Conclusion: Occult and clinically irrelevant CSF leaks do occur in a significant proportion of patients during and shortly after FESS. Intra- and postoperatively, routine $\beta 2$-transferrin assays show low sensitivity. They should not be used in these settings. The clinical course of patients with occult CSF leaks indicated possibility of an uneventful follow-up.

Key words: CSF leak, FESS, $\beta 2$-transferrin, $\beta$-trace protein, sinus surgery

\section{Introduction}

Surgery of the paranasal sinuses is a common intervention in rhinology for the treatment of chronic inflammation, tumours and other pathologies. These interventions are performed in close vicinity to the skull base. In this context, a cerebrospinal fluid (CSF) leak, due to disruption of the anterior skull base meninges, represents a severe complication ${ }^{(1-7)}$. A CSF leak bears the potential risk of bacterial meningitis, intracerebral hypotension and pneumocephalus ${ }^{(1,8)}$. Therefore, it is important to detect the incidence of CSF leaks after functional endoscopic sinus surgery (FESS). In the bloody operation field, the emersion of the clear CSF is not always obvious ${ }^{(1,8)}$. Its detection is difficult and leads to late diagnosis ${ }^{(1)}$ : CSF can be mimed by watery rhinorrhea in the context of chronic rhinosinusitis or post-injury autonomic dysfunction ${ }^{(8)}$. Furthermore, contamination of the samples collected from nasal cavities may occur by clear nasal mucus or blood ${ }^{(8)}$. Therefore, methods to detect CSF traces are essential. These methods should be non-invasive, easy to perform with routine laboratory equipment, should not involve radiation and should require only small quantities of samples to be assessed. Hence, $\beta 2$-transferrin assays are the first line method for detecting CSF leaks ${ }^{(9)}$ and are routinely used ${ }^{(1,9)}$, even though $\beta$-trace protein assays are described to be more accurate ${ }^{(8,10)}$. However, the diagnostic performance of both assays 
is rather controversial in blood-contaminated settings $(6,9,11)$ as it is the case after FESS. In this study we assessed the performance of the assays and determined the incidence of occult CSF leaks. Since it is not known whether an occult CSF leak will eventually lead to a clinically symptomatic and permanent leak ${ }^{(10)}$, we also aimed at assessing whether patients with an occult leak directly after the operation still had a positive test at least one year after surgery.

\section{Subjects and methods}

We performed a monocentric prospective cohort study of patients undergoing standard FESS procedures ${ }^{(12)}$ of at least one sinus at the Department of Otorhinolaryngology, Head and Neck Surgery, University Hospital of Zurich. The patients were recruited the day before surgery and written informed consent was obtained after thorough information. The following data were collected by paper CRF (Case Report Form) including age at time of surgery, date of surgery, indication, performed operation, surgeon, results of the $\beta 2$-transferrin assay on each operated side, the follow up after 2 weeks: symptomatic CSF leak yes/no and the results of the reanalysis of the CSF positive patients after $>1$ year by the $\beta$-trace protein assay. At the end of the surgery small $25 \times 50 \mathrm{~mm}$ patties were placed into both middle meatus under endoscopic guidance, followed by standard Netcell ${ }^{\circledR}$ packing. The $2 \mathrm{~mm}$ thin Neuray ${ }^{\circledR}$ or Vostra ${ }^{\circledR}$ gauze is highly absorptive and is routinely used in ear, nose and throat as well as neurosurgery for the detection of CSF. It was removed together with the packing a day after surgery and was compared with a venous blood sample $(8 \mathrm{ml})$ for detection of CSF by means of $\beta 2$-transferrin testing. The comparison of blood and rhinorrhea samples reduced false positive results, due to the general expression of $\beta 2$-transferrin in the serum of some patients ${ }^{(8)}$. The volume of the analysed secretions was $10 \mu \mathrm{l}$ and blood sampling took place during general anaesthesia. Samples were frozen, stored and analysed later with the intention to reduce costs. The first analysis comprised a qualitative, visual inspection of the electrophoretic pattern by using the hydrogel $6 \beta 2$ transferrin (SEBIA, France) assay according to the manufacturer's instructions. In a second step, a more quantitative reanalysis by densitometric scanning was performed. The detection of CSF with the $\beta 2$-transferrin assay, as defined by the manufacturer (2-asialtransferrin/0-asialtransferrin $>1$ ), was complicated by the inevitable contamination of the samples with blood. Finally, we defined a sample as positive for CSF, if there was a clear difference in the fraction of 2-asialtransferrin and 0-asialtransferrin (total transferrin) when comparing the two sides in the same patient. A difference was regarded as clear, if one side showed more than four times the value for the fraction of 2-asialtransferrin and 0 -asialtransferrin than the other side. Due to this approach, the detection of small amounts of CSF was possible. Two weeks after surgery, routine follow-up was performed: The patients were specifically inquired for clear rhinorrhea. The study protocol foresaw that if clear discharge was present, a reanalysis of $\beta 2$-transferrins and a CT of the paranasal sinuses would take place. For study purposes, patients with CSF positive testing were reanalysed by a $\beta$-trace protein assay after $>1$ year, if the patient reported any kind of nasal discharge, although they were otherwise asymptomatic. Additional follow-up visits outside of the study were allowed at any point of time, i.e. for cleaning the nasal cavity.

We evaluated the minimal detection level of CSF by $\beta 2$-transferrin and $\beta$-trace protein assays in the blood-contaminated setting by a post-hoc-analysis of an own titration. For that purpose, we used serum samples with defined CSF concentrations. The analysis of our titration was performed at another institution (Kantonsspital Aarau) ensuring the blinding of the CSF concentrations in samples. The $\beta 2$-transferrin assay was analysed by electrophoresis and densitometric scanning and the $\beta$-trace protein assay by nephelometry. The results of the $\beta 2$-transferrin in-vitro titration were verified by the $\beta 2$-transferrin assay with electrophoretic and densitometric scanning in a patient with a primary spontaneous CSF leak.

\section{Exclusion criteria}

In case of an accidental injury of the skull base during surgery, a neoplasm at the skull base or a pre-existing CSF leak, the patient would have been excluded from the study. Additionally, we intended to exclude patients where intraoperative CSF leak would have been found and sealed. However, none of these complications occurred during the study period.

\section{Number of cases}

The number of patients was planned to be able to detect at least a $1 \%$ occurrence of an occult CSF leak. Therefore, a maximum of 50 patients with bilateral testing was foreseen. Due to an interim analysis with already positive results in more than $5 \%$, the study was stopped after 30 patients (57 samples). The study population included 2 primary revision cases as well as 9 multiple revision cases ( 3 to 5 surgeries with a median of 4). Furthermore, there were four adverse events due to minor bleeding upon removal of the neurosurgical patty. Hence, the study group felt it was unethical to continue the study in view of already present positive results.

\section{Statistical methods}

The study's primary endpoint was the positive $\beta 2$-transferrin assay result in order to detect the reassured incidence of occult CSF leaks after FESS. Differences between the surgeons and surgeries were investigated by the chi-square test.

\section{Ethics}

This study was approved by the Swiss Ethics Committees (KEK- 
Table 1. Subject characteristics.

\begin{tabular}{|c|c|c|}
\hline Characteristics & Subclassification & Numbers (\%) \\
\hline Male / female $(n=30)$ & & $\begin{array}{l}17 / 13 \\
(57 \% / 43 \%)\end{array}$ \\
\hline Mean age (years) & & $41.4 \pm 13.7$ \\
\hline Diagnosis $(n=30)$ & $\begin{array}{l}\text { CRSwNP } \\
\text { raRS } \\
\text { CRSsNP }\end{array}$ & $\begin{array}{l}17(56.7 \%) \\
8(26.7 \%) \\
5(16.7 \%)\end{array}$ \\
\hline $\begin{array}{l}\text { Surgery types } \\
(n=57)\end{array}$ & $\begin{array}{l}\text { spheno-ethmoidectomy } \\
\text { total ethmoidectomy } \\
\text { fronto-ethmoidectomy } \\
\text { anterior ethmoidectomy } \\
\text { fronto-spheno-ethmoi- } \\
\text { dectomy } \\
\text { infundibulotomy }\end{array}$ & $\begin{array}{l}23(40.4 \%) \\
11(19.3 \%) \\
11(19.3 \%) \\
5(8.8 \%) \\
5(8.8 \%) \\
2(3.5 \%)\end{array}$ \\
\hline $\begin{array}{l}\text { Revision surgeries } \\
(n=57)\end{array}$ & & $11(19.3 \%)$ \\
\hline $\begin{array}{l}\text { Skull base identified } \\
(n=57)\end{array}$ & & $47(82.5 \%)$ \\
\hline $\begin{array}{l}\text { Mean time of surgery } \\
\text { (min) }\end{array}$ & & $70.8 \pm 28.6$ \\
\hline
\end{tabular}

CRSwNP: chronic rhinosinusitis with polyps, CRSsNP: chronic rhinosinusitis without polyps, raRS: recurrent acute rhinosinusitis

2014-0678) on research involving humans. It was conducted in compliance with formalities of the independent ethical commission, the current Helsinki-Declaration as well as the Swiss law.

\section{Results}

The male/female ratio of the study population $(n=30)$ was $17 / 13$ and the average age was $41.4 \pm 13.7$ years. Further demographic information is presented in Table 1. An intraoperative CSF leak was not observed. The rhinorrhea samples from nasal tamponades were all blood-contaminated to some extent. The rhinorrhea samples were collected unilaterally in three and bilaterally in 27 patients. The results of the hydrogel $6 \beta 2$-transferrin assay are given in Table 2.

\section{Occult CSF leak}

Four out of the 30 patients were positive for CSF on one side - resulting in an overall CSF microleak rate of 13\% after FESS. When counting the 57 operated sides separately, 4/57 samples were positive (7\%). None of the primary or multiple revision cases were positive for CSF. The CSF positive patients with any sign of spontaneous nasal discharge after more than one year after surgery (3/4) were retested between 22 and 23 months after surgery, respectively. In the follow-up, these patients showed negative results in a bilateral (mono-nostril) $\beta$-trace protein assay and none of them developed clinically relevant CSF rhinorrhea or meninigitis. None of the other parameters revealed to be a significant risk factor for CSF positive samples.
Table 2. Characteristics of positive samples*

\begin{tabular}{|c|c|c|}
\hline Characteristics & Subclassification & Numbers (\%) \\
\hline $\begin{array}{l}\text { Positive/negative } \\
\text { CSF samples }\end{array}$ & & 4/53 (7\%/93\%) \\
\hline Male/female $(n=4)$ & & $1 / 3(25 \% / 75 \%)$ \\
\hline Surgery types $(n=4)$ & $\begin{array}{l}\text { fronto-ethmoidectomy } \\
\text { anterior ethmoidectomy } \\
\text { fronto-spheno-ethmoi- } \\
\text { dectomy }\end{array}$ & $\begin{array}{l}2(50 \%) \\
1(25 \%) \\
1(25 \%)\end{array}$ \\
\hline Diagnosis $(n=4)$ & $\begin{array}{l}\text { CRSwNP } \\
\text { raRS }\end{array}$ & $\begin{array}{l}2(50 \%) \\
2(50 \%)\end{array}$ \\
\hline $\begin{array}{l}\text { Skull base identified } \\
(n=4)\end{array}$ & & $4(100 \%)$ \\
\hline Age (years) & & 25 to 66 \\
\hline
\end{tabular}

* under exclusion of the positive control sample.

Abbreviations: CSF: cerebrospinal fluid, CRSwNP: chronic rhinosinusitis with polyps raRS: recurrent acute rhinosinusitis.

The distribution of positive findings across different operations is shown in Figure 1.

\section{Detection level of CSF in the blood-contaminated setting}

Post-hoc-analysis of own titration showed that the $\beta$-trace protein assay is more sensitive in detecting CSF compared to the $\beta 2$-transferrin assay in the blood-contaminated setting. The former is able to detect CSF at a minimum threshold of 5\% CSF and the latter of $20 \%$ in strongly serum-contaminated rhinorrhea samples. The $\beta 2$-transferrin assay performed in a sample obtained from a patient with a primary spontaneous CSF leak confirmed these findings, while being negative when using the manufacturer's cut-off values and only turning positive with the above-mentioned adaptations of analysis methods.

\section{Discussion}

This study provides two important new findings concerning CSF leaks after FESS and its testing by $\beta 2$-transferrin assay. First, occult CSF leaks do occur in a relevant proportion of patients during or shortly after FESS. These leaks are clinically irrelevant, since all cases showed spontaneous resolution and an uneventful course. Second, routine $\beta 2$-transferrin assays show low sensitivity in blood-contaminated samples and should not be used in this setting. If no other option is available, we suggest using a diagnostic approach as it is described in this study by the comparison of the two sample sides regarding their 2-asialtransferrin/0-asialtransferrin ratios in a densitometric analysis. Based on own titration and the literature, we suggest the $\beta$-trace protein assay as being a superior alternative in these conditions.

We detected an incidence of $13 \%$ for occult CSF leaks after FESS, 


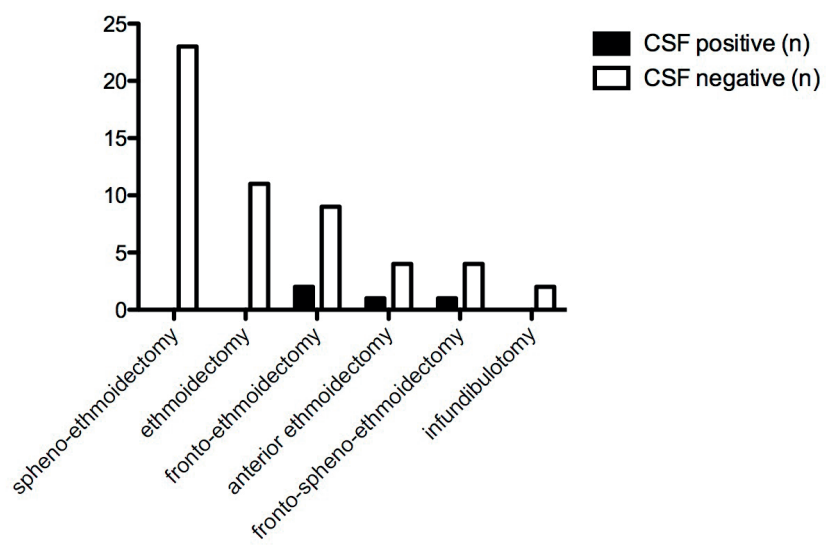

Figure 1. Occult CSF leaks in different types of surgeries.

which is higher compared to previous studies ${ }^{(10,13)}$. One of them found an incidence of $2.9 \%$ for occult CSF leaks after FESS by a $\beta$-trace protein assay ${ }^{(10)}$. In other studies, CSF positive samples after FESS were never detected using $\beta$-trace protein ${ }^{(13)}$ and $\beta 2$ transferrin assays ${ }^{(14)}$. These diverging results could be explained by several factors. The study of Bachmann-Harildstad et al. performed less radical surgical treatments under local anaesthesia ${ }^{(13)}$. Solomon et al. were probably confronted with the same difficulty of CSF detection in blood-contaminated settings using a $\beta 2$-transferrin assay ${ }^{(14)}$. By applying the manufacturer's threshold of $>1$ for the fraction of 2-asialtransferrin/0-asialtransferrin, we experienced that this method shows no positive results for CSF in blood-contaminated samples. Therefore, and in contrast to the study of Solomon et al., we adjusted the diagnostic criteria comparing the results of both sides in one patient in order to account for the postoperatively blood-stained field as described above.

The course of occult CSF leaks after FESS is not explored in literature, but it lets us suppose that intraoperative occult CSF leaks might occlude spontaneously during the postoperative healing process ${ }^{(10)}$. Furthermore, spontaneous closures of e.g. posttraumatic lesions in the dura mater are well known. Considering that post-traumatic CSF rhinorrhea results in meningitis early in its course and only if CSF leaks are identified by positive testing (15), our findings are consistent with this assumption. The course of all retested patients was also uneventful and all tests negative for CSF in the follow-up after > 1 year. Nonetheless, we would be cautious and advice a close, long-term follow-up of patients with a confirmed CSF leak, who are symptomatic, for evaluation of a selective intervention when needed.

The rate of occult CSF leaks seemed not to be higher in the different groups formed by the type of surgery, the duration of surgery, the experience of the surgeon, the indication for surgery, the age or patients with polyps compared to the group without. Without doubt, this might be due to low sample size and a low incidence of occult CSF leaks and could be explored in a bigger sample of patients.

Our study also addressed the problem of CSF detection in blood-contaminated settings. Leaking CSF can be detected by several methods: 1) electrophoretic examination of nasal fluid for the presence of $\beta 2$-transferrin; 2 ) immunonephelometry measuring $\beta$-trace protein concentration; 3 ) high resolution $C T$ or 4) magnetic resonance (MR) cisternography with contrast media; and 5) surgical exploration ${ }^{(16-22)}$. While invasive imaging diagnostics are accurate in the detection of CSF leaks, they will only detect large leaks, are expensive, and carry the risk of inducing a chemical meningitis due to the intrathecal contrast dye injection ${ }^{(9)}$. Therefore, $\beta 2$-transferrin assays have been suggested as first line method for detecting CSF leaks and are used routinely ${ }^{(9)}$. Under non-contaminated conditions, these assays have an excellent sensitivity, specificity, are non-invasive and inexpensive ${ }^{(23,24)}$. However, their diagnostic performance in blood-contaminated samples is rather controversial ${ }^{(6,9,11)}$. Hence, many studies used blood-contaminated samples as an exclusion factor ${ }^{(6,9)}$. However, in the postoperative setting (but also after other head injuries) - to a greater or lesser extent - all samples are blood-contaminated. Our study reassured that the interpretation of blood-contaminated samples by using routine $\beta 2$-transferrin assay is misleading and their sensitivity and specificity in the postoperative setting is probably not appropriate. According to manufacturers' instructions, the hydrogel $6 \beta 2$ transferrin assay is able to detect CSF until a minimal level of $4 \%$ CSF in rhinorrhea. We would like to point out that this detection limit could not be verified in this study, neither with electrophoretic nor with densitometric analyses. Measurements in our own laboratory by $\beta 2$-transferrin assay with electrophoretic and densitometric analysis showed that already a mixture of $80 \%$ serum and $20 \%$ CSF can be difficult to be evaluated as positive. By applying the diagnostic criteria of the manufacturer for CSF positive samples, our in-vivo verification sample obtained from a primary CSF leak was negative for CSF because of the bloodcontamination intra- and postoperatively. Besides the difficulty in detecting CSF in blood-contaminated samples, there is also the risk of false positive results. Elevated plasmatic $\beta 2$-transferrin levels (for example in case of chronic alcoholism, severe liver disease, glycoprotein metabolism disorders or genetic variations of $\beta 2$-transferrin) can lead to false-positive results ${ }^{(25,26)}$. This problem can be solved by running the patient's serum in parallel to the rhinorrhea sample and comparing the asialotransferrin/ disialotransferrin bands ratio in the two samples using densitometric scanning ${ }^{(8)}$. Another problem are false positive samples in the presence of haemoglobin, which could have been released during surgery ${ }^{(9)}$. However, if $\beta 2$-transferrin assays are performed on peri- or early postoperative patients, they will very likely observe a clinical irrelevant leak. This would result in a false positive test, which could lead to further interventions (e.g. attempts to close that leak ${ }^{(15)}$ ) causing more harm than 
good. Based on the resulting low sensitivity and specificity of the assay, we suggest avoiding the use of $\beta 2$-transferrin assays for detecting CSF after FESS. When no other option is available, we suggest using the diagnostic approach as it is described in this study by the comparison of the two sample sides regarding their 2-asialtransferrin/0-asialtransferrin ratios in a densitometric analysis.

The $\beta$-trace protein assay is a valuable alternative compared to routine $\beta 2$-transferrin assays in postoperative or blood-contaminated conditions. While the $\beta$-trace protein assay seems to have similar analytical sensitivity under non-contaminated conditions as the $\beta 2$-transferrin assay ${ }^{(9)}$, it shows a clear advantage in detecting CSF in blood-contaminated samples. Our titration showed a detection limit of $\beta$-trace protein approximately at 0.5 $\mathrm{mg} / \mathrm{l}$ or $<5 \%$ CSF in the blood-contaminated setting, which is in consensus with others ${ }^{(8,10)}$. In some studies, the detection limit of $\beta$-trace protein in CSF is controversially discussed and ranged from $0.35 \mathrm{mg} / \mathrm{l}$ to $6 \mathrm{mg} / \mathrm{l}^{(11,27)}$. Apparently, these discrepancies were due to the heterogeneous nature of samples tested and whether samples were analysed after being diluted or not ${ }^{(9)}$. Based on our findings, we suggest the use of the $\beta$-trace protein assay in blood-contaminated samples, while keeping in mind that even this assay has limitations such as renal insufficiency or bacterial meningitis which both can have an impact on sensitivity $^{(28)}$ as well as showing irrelevant CSF leaks as reported by our study.

Our study has limitations. We did not obtain $\beta 2$-transferrin testing before surgery, which is a shortcoming in this study that could lead to false interpretation of results due to either pre-existing CSF rhinorrhea or false positive results. However, false positive test results are considered to be infrequent and spontaneous CSF leaks are even less common. When running CSF and serum in parallel, false positive readings of $\beta 2$-transferrin assays are highly unlikely ${ }^{\left({ }^{8}\right)}$, especially when comparing sides within one individual. Furthermore, if pre-existing rare primary or secondary CSF rhinorhea was present before surgery, it would probably not cease spontaneously in the postoperative course, which was clearly shown by our long-term follow-up $\beta$-trace testing.

It was our primary concern to measure the incidence of occult CSF leaks after FESS and according to the existing literature we chose the $\beta 2$-transferrin assay as the primary detection method.
As shown in this study, this assay has limitations in bloodcontaminated samples. Therefore, we performed a post-hoc analysis for the $\beta 2$-transferrin assay and compared the two sides of the nasal cavities. By doing this, we could find an incidence of occult CSF leaks that is in line with others. A definite comparison between the reliability of $\beta 2$-transferrin and $\beta$-trace protein assays, however, cannot be asserted by our study as it was not foreseen in the study design. Due to this fact and to investigate whether there are other factors influencing the incidence of occult CSF leaks after FESS, further studies would be needed.

\section{Conclusion}

In conclusion, occult, but also clinically irrelevant CSF leaks do occur in a significant proportion of patients during or shortly after FESS. In our study, the incidence of occult CSF leaks after FESS was $13 \%$ and therefore higher compared to previous studies. $\beta 2$-transferrin assays show low accuracy in bloodcontaminated samples intra- and postoperatively and should not be used in this setting. Based on our own titration and the literature, we suggest the $\beta$-trace protein assay being a superior alternative in this condition. However, our study showed that the surgeon must be aware of the possibility of an occult CSF leak after FESS, especially when performing postoperative CSF leak testing. All results discourage from testing potential CSF leaks in the early postoperative setting, due to the risk of irrelevant positive or false negative results, in clinically asymptomatic patients.

\section{Acknowledgement}

Cantonal Ethics Committee Zurich, No.: 2014-0678

\section{Authorship contribution}

SB has written the manuscript and performed result analyses; AK has recruited the patients and corrected the manuscript; EP has performed all laboratory analyses; LE has done statistical testing and helped writing the manuscript; RRS has planned the study; DH has collected samples and corrected the manuscript; MBS has planned and supervised the study, collected samples and helped writing the manuscript.

\section{Conflict of interest}

The authors declare no conflict of interest.

\section{References}

1. Kubik M, Lee S, Snyderman C, Wang E. Neurologic sequelae associated with delayed identification of iatrogenic skull base injury during endoscopic sinus surgery (ESS). Rhinology. 2017; 55: 53-58.

2. Tomazic PV, Stammberger $H$, Koele W Gerstenberger C. Ethmoid roof CSF-leak following frontal sinus balloon sinuplasty. Rhinology. 2010; 48: 247-250.
3. Schnabel C, Di Martino E, Gilsbach JM, Riediger D, Gressner AM, Kunz D. Comparison of beta2-transferrin and betatrace protein for detection of cerebrospinal fluid in nasal and ear fluids. Clin Chem. 2004; 50: 661-663.

4. Grevers G. Anterior skull base trauma during endoscopic sinus surgery for nasal polyposis preferred sites for iatrogenic injuries. Rhinology. 2001; 39: 1-4
5. Kerr JT, Chu FW, Bayles SW. Cerebrospinal fluid rhinorrhea: diagnosis and management. Otolaryngol Clin North Am. 2005; 38: 597-611.

6. Gorogh T, Rudolph P, Meyer JE, Werner JA, Lippert BM, Maune S. Separation of beta2transferrin by denaturing gel electrophoresis to detect cerebrospinal fluid in ear and nasal fluids. Clin Chem. 2005; 51: 1704-1710.

7. Papadea C, Schlosser RJ. Rapid method for 
beta2-transferrin in cerebrospinal fluid leakage using an automated immunofixation electrophoresis system. Clin Chem. 2005; 51: 464-470.

8. Lescuyer $P$, Auer L, Converset $V$, Hochstrasser DF, Landis BN, Burkhard PR. Comparison of gel-based methods for the detection of cerebrospinal fluid rhinorrhea. Clin Chim Acta. 2012; 413: 1145-1150.

9. McCudden CR, Senior BA, Hainsworth $S$ Oliveira W, Silverman LM, Bruns DE, et al. Evaluation of high resolution gel beta2transferrin for detection of cerebrospinal fluid leak. Clin Chem Lab Med. 2013; 51: 311-315.

10. Bachmann $G$, Djenabi $U$, Jungehulsing $M$ Petereit $\mathrm{H}$, Michel O. Incidence of occult cerebrospinal fluid fistula during paranasal sinus surgery. Arch Otolaryngol Head Neck Surg. 2002; 128: 1299-1302.

11. Reiber $H$, Walther $K$, Althaus $H$. Beta-trace protein as sensitive marker for CSF rhinorhea and CSF otorhea. Acta Neurol Scand. 2003; 108: 359-362.

12. Soyka MB, Holzmann D. Correlation of complications during endoscopic sinus surgery with surgeon skill level and extent of surgery. Am J Rhinol. 2005; 19: 274-281.

13. Bachmann-Harildstad G. Incidence of CSF fistula after paranasal sinus surgery: the Northern Norwegian experience. Rhinology. 2007; 45: 305-307.

14. Solomon P, Chen J, D'Costa M, Gilbert R, Davidson J, Johnston M. Extracranial drainage of cerebrospinal fluid: a study of betatransferrins in nasal and lymphatic tissues. Laryngoscope. 1999; 109: 1313-1315.

15. Poletti-Muringaseril SC, Rufibach K, Ruef C, Holzmann D, Soyka MB. Low meningitisincidence in primary spontaneous com- pared to secondary cerebrospinal fluid rhinorrhoea. Rhinology. 2012; 50: 73-79.

16. Bachmann G, Nekic M, Michel O. Clinical experience with beta-trace protein as a marker for cerebrospinal fluid. Ann Oto Rhinol Laryngol. 2000; 109: 1099-1102.

17. Burkhard PR, Rodrigo N, May D, Sztajzel R, Sanchez JC, Hochstrasser DF, et al. Assessing cerebrospinal fluid rhinorrhea: a two-dimensional electrophoresis approach. Electrophoresis. 2001; 22: 1826-1833.

18. Marshall AH, Jones NS, Robertson IJ. An algorithm for the management of CSF rhinorrhoea illustrated by 36 cases. Rhinology. 1999; 37: 182-185.

19. Meco C, Oberascher G. Comprehensive algorithm for skull base dural lesion and cerebrospinal fluid fistula diagnosis. Laryngoscope. 2004; 114: 991-999.

20. Payne RJ, Frenkiel S, Glikstein R, Mohr G. Role of computed tomographic cisternography in the management of cerebrospinal fluid rhinorrhea. J Otolaryngol. 2003; 32: 93-100

21. Zapalac JS, Marple BF, Schwade ND. Skull base cerebrospinal fluid fistulas: a comprehensive diagnostic algorithm. Otolaryngol Head Neck Surg. 2002; 126: 669-676.

22. Sanders EL, Clark RJ, Katzmann JA Cerebrospinal fluid leakage: agarose gel electrophoresis detection of beta(2)-transferrin and nephelometric quantification of beta-trace protein. Clin Chem. 2004; 50: 2401-2403.

23. Grantham VV, Blakley B, Winn J. Technical review and considerations for a cerebrospinal fluid leakage study. J Nucl Med Technol. 2006; 34: 48-51.

24. Normansell DE, Stacy EK, Booker CF, Butler TZ. Detection of beta-2 transferrin in otor rhea and rhinorrhea in a routine clinical laboratory setting. Clin Diagn Lab Immunol. 1994; 1: 68-70.

25. Sloman AJ, Kelly RH. Transferrin allelic variants may cause false positives in the detection of cerebrospinal fluid fistulae. Clin Chem. 1993; 39: 1444-1445.

26. Roelandse FW, van der Zwart N, Didden JH, van Loon J, Souverijn JH. Detection of CSF leakage by isoelectric focusing on polyacrylamide gel, direct immunofixation of transferrins, and silver staining. Clin Chem. 1998; 44: 351-353.

27. Bachmann G, Petereit $H$, Djenabi U, Michel O. Predictive values of beta-trace protein (prostaglandin D synthase) by use of lasernephelometry assay for the identification of cerebrospinal fluid. Neurosurgery. 2002; 50: 571-576; discussion 6-7.

28. Meco C, Oberascher G, Arrer E, Moser G, Albegger K. Beta-trace protein test: new guidelines for the reliable diagnosis of cerebrospinal fluid fistula. Otolaryngol Head Neck Surg. 2003; 129: 508-517.

Michael Soyka, MD

Department for ENT Surgery

University Hospital Zurich

Frauenklinikstrasse 24

$\mathrm{CH}-8091$ Zürich

Switzerland

Tel: +41 442551111

E-mail:michael.soyka@usz.ch 